\title{
FLIGHT TESTS OF THE DELICAT AIRBORNE LIDAR SYSTEM FOR Remote Clear Air Turbulence Detection
}

\author{
Patrick Vrancken $^{1 *}$, Martin Wirth ${ }^{1}$, Gerhard Ehret ${ }^{1}$, Benjamin Witschas ${ }^{1}$, Henk Veerman ${ }^{2}$, \\ Robert Tump ${ }^{2}$, Hervé Barny ${ }^{3}$, Philippe Rondeau ${ }^{3}$, Agnès Dolfi-Bouteyre ${ }^{4}$, Laurent Lombard ${ }^{4}$ \\ ${ }^{I}$ Deutsches Zentrum für Luft- und Raumfahrt (DLR) - German Aerospace Center, Institute of \\ Atmospheric Physics, Oberpfaffenhofen, 82234 Weßling, Germany, *Email: patrick.vrancken@dlr.de \\ ${ }^{2}$ National Aerospace Laboratory (NLR), Anthony Fokkerweg 2, 1059CM Amsterdam, The Netherlands \\ ${ }^{3}$ THALES Avionics, 25 Rue Jules Védrines, 26027 Valence Cedex, France \\ ${ }^{4}$ ONERA - The French Aerospace Lab, 91123 Palaiseau, France
}

\begin{abstract}
An important aeronautics application of lidar is the airborne remote detection of Clear Air Turbulence which cannot be performed with onboard radar. We report on a DLR-developed lidar system for the remote detection of such turbulent areas in the flight path of an aircraft. The lidar, consisting of a high-power UV laser transmitter and a direct detection system, was installed on a Dutch research aircraft. Flight tests executed in 2013 demonstrated the performance of the lidar system to detect local subtle variations in the molecular backscatter coefficient indicating the turbulence some 10 to $15 \mathrm{~km}$ ahead.
\end{abstract}

\section{INTRODUCTION}

In commercial aviation, Clear Air Turbulence (CAT) encounter is a leading cause for injuries to cabin crew and passengers causing $\mathrm{M} \$$ per year to airlines. Occurring at cruise altitudes in clear air, CAT may not be detected by radar due to the absence of hydrometeors. Active optical sensing with lidar is the only possible means of remotely detecting CAT. Following detection, a warning to cabin crew and passengers may be issued or a mitigation action initiated (deceleration or evasion maneuver, e.g.). Generally, lidar detection is possible with different methods, by either measuring wind speed fluctuations with coherent Doppler wind lidar or air density fluctuations, the latter method described herein. Despite its apparent suitability, coherent Doppler lidar has the disadvantage of relying on backscatter from aerosols that are, at cruise flight altitudes, not sufficiently present for a generally reliable and long-range detection of CAT. Second, it measures wind speed in the direction of line-of-sight whereas the hazardous turbulent winds occur vertically, thus perpendicular to the flight path. Due to anisotropy of the turbulence, these horizontal and vertical wind speed fluctuations are not the same, and thus the turbulence strength may not be determined reliably.

Another method to measure CAT is proposed in [1], and a technology demonstration activity, funded by the European Commission aeronautics research programme, is described in [2]. The detection principle relies on tiny fluctuations of air temperature and thus density when air parcels undergo the up- and downwelling motion in the buoyancy range of the turbulence. Under CAT conditions, a simple relationship between vertical wind speed, air density $\rho_{\text {air }}$ and hence backscatter coefficient $\beta_{\pi, m o l}$ fluctuation may be derived [2]:

$$
\sigma_{w}=\frac{g}{N} \cdot\left(\frac{\partial \rho_{\text {air }}}{\rho_{\text {air }}}\right)=\frac{g}{N} \cdot\left(\frac{\partial \beta_{\pi, m o l}}{\beta_{\pi, m o l}}\right)
$$

where $\sigma_{w}$ is the vertical wind speed turbulent fluctuation, $g$ the gravity acceleration, and $N$ the stratification stability that may be derived sufficiently well from numerical weather forecast (or determined in-situ). For a practical lidar application, a strong molecular backscatter is thus crucial and may be achieved with a UV wavelength. In the described approach within the European DELICAT project [2], a high power UV laser beam is aimed into the flight direction and the backscattered radiation is sufficiently averaged in order to attain the necessary level of resolution, which is on the percent level for the "moderate" turbulence. In the following, this lidar system is described, an overview of the flight campaign is given and the measurement results are discussed. 


\section{LIDAR SYSTEM}

The lidar system for the detection of the small turbulence-induced air density fluctuations was designed by DLR and is comprised of the following elements (a synopsis is given in Fig. 1): The transmitter is based on the high-power Nd:YAG system of the WALES lidar [3].

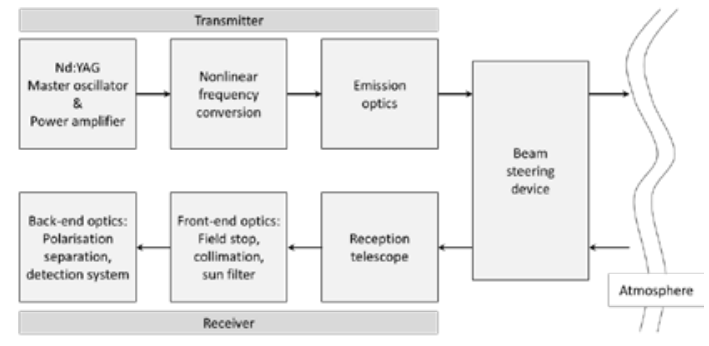

Figure 1: Synopsis of the DELICAT lidar system

A single-mode MOPA monolithic ring laser is locked to an Iodine absorption cell and subsequently amplified in three linear power amplifiers. Its linearly polarized short Q-switched infrared pulses are frequency converted to green and subsequently to UV by sum-frequency generation. Technical details are given in Table 1.

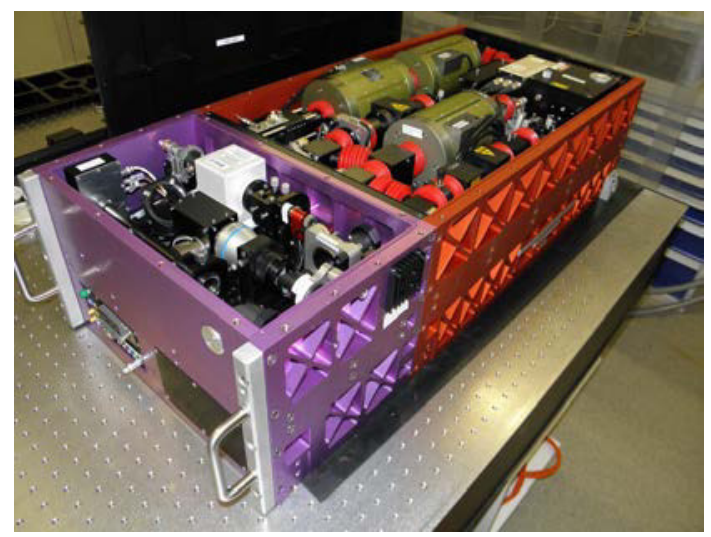

Figure 2: Transmitter: ND:YAG laser and THG.

The transmit laser beam is then guided onto a beam steering system, developed by THALES. This system ensures that the laser beam is always pointed into flight direction, no matter what the attitude of the aircraft (due to angle of attack for a chosen flight speed, mass, etc.). The system, comprised of two moving and one fixed mirror (the latter on the outside of the aircraft fuselage) tracks the aircraft movements by reading the ARINC data from the aircraft's inertial platform (IRS) and a measurement boom. The laser beam is thus guided, over the mirrors and some windows to the outside of the aircraft and directed into the flight path direction.

The receiver system (monostatic architecture regarding the transmit beam), consists of a $140 \mathrm{~mm}$ Newtonian telescope, spatial and frequency filters, a polarisation separation assembly and two PMT detection devices.

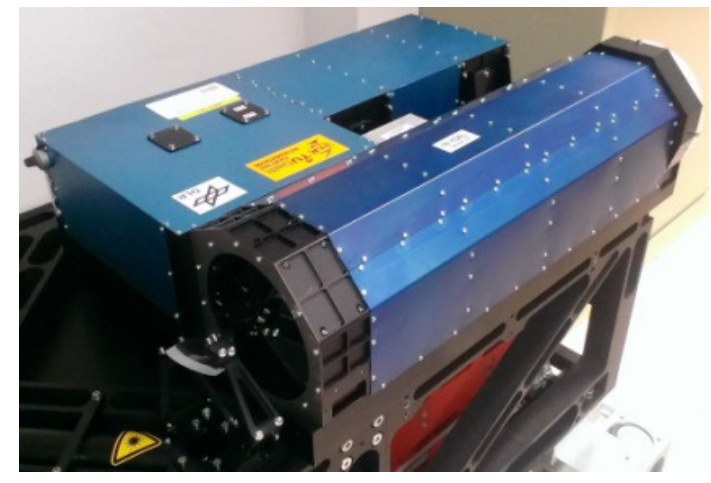

Figure 3: Receiver system.

The polarisation separation assembly allows the measurement of the depolarisation due to aerosols (the molecular depolarisation amounts to approximately $0.5 \%$ under the respective conditions). This depolarisation would give an indication of spurious aerosol backscatter, admittedly not systematically, but at least for Cirrus ice crystals and the like. A spectral separation with a high resolution spectral filter (such as a Fabry-Perot interferometer, e.g.) is planned for an upcoming upgraded version of the lidar. The present receiving system further allows, due to its modular layout, the versatile testing of various detection schemes for other aeronautics applications, such as volcanic ash or hazardous crystalline icing conditions detection.

The PMT detectors' read-out is digitized with a selectable sample rate, $30 \mathrm{MHz}$ (i.e. a range resolution of $5 \mathrm{~m}$ ) has been chosen for the present tests. The detectors, as well as the data acquisition system (also controlling the transmitter) are likewise issued from the WALES lidar [3].

The lidar system elements, together with the beam steering system are integrated into a stiff rack which is in turn mounted onto the test aircraft's seat rails. Apart from the lidar, a two-circuit cooling system has been developed, evacuating the laser thermal discharge to the outside air and thus preventing the heating-up of the small cabin of the research aircraft. 


\begin{tabular}{|c|c|}
\hline Parameter & Value \\
\hline Repetition rate & $100 \mathrm{~Hz}$ \\
\hline Laser pulse energy (IR) & $400 \mathrm{~mJ}$ \\
\hline Laser pulse energy (UV) & $85 \mathrm{~mJ}$ \\
\hline Line width (IR) & $54 \mathrm{Mhz}$ \\
\hline Frequency stability (IR) & $\leq 1 \mathrm{MHz}$ \\
\hline Pulse length & $\overline{8} \mathrm{~ns}$ \\
\hline Beam quality $\mathrm{M}^{2}(\mathrm{UV})$ & 4.3 \\
\hline Beam divergence & $150 \mu \mathrm{rad}$ \\
\hline Telescope diameter & $140 \mathrm{~mm}$ \\
\hline Field of view & $530 \mu \mathrm{rad}$ \\
\hline Sun filter bandwidth (FWHM) & $0.5 \mathrm{~nm}$ \\
\hline System depolarisation & $0.9 \%$ \\
\hline Detector NEP & $0.25 \mathrm{fW} / \sqrt{ } \mathrm{Hz}$ \\
\hline Analog/digital converter & 14 bit \\
\hline Sampling rate & $30 \mathrm{MHz}(5 \mathrm{~m})$ \\
\hline Beam steering dynamic & $1 \mathrm{~Hz}$ (sine) \\
\hline Pointing accuracy & $0.1^{\circ}$ \\
\hline
\end{tabular}

\section{TEST AIRCRAFT AND FLIGHT CAMPAIGN}

After ground test at Oberpfaffenhofen, Germany, the lidar system was installed on the NLR's Cessna Citation 2 aircraft. It offers a maximum ceiling of $43,000 \mathrm{ft}$ at maximum cruise speed of $200 \mathrm{~m} / \mathrm{s}$. The aircraft was fitted with an aerodynamic fairing covering the external mirror that bends the laser beam to the front (see Fig. 4). Further, it was equipped with an external liquid cooling system (jointly developed by NLR and DLR), an inertial reference system (IRS), a noseboom measuring side-slip angle, and a fast temperature sensor. As a truth for lidar-detected turbulent zones ahead could thus be used the vertical acceleration (from IRS) - the response to vertical wind speed fluctuation $\sigma_{w}-$ and the temperature fluctuations, both when the aircraft is travelling through the probed area some seconds after lidar detection.

All modifications on the aircraft, on the fuselage, in the cabin, related to procedures and to laser safety within and outside the aircraft have been subject to airworthiness certification. An STC (Supplemental Type Certificate) was issued to the aircraft PH-LAB with the DELICAT modifications in July, 2013 by Dutch CAA.

The flight test campaign [4] was performed in July and August 2013 from Amsterdam Schiphol airport. The flight test crew of NLR, together with lidar operators of DLR have been assisted by
Warsaw University and Météo France, procuring aeronautical weather forecast and different CAT forecast metrics. Another task was to identify areas "devoid" of aerosols and Cirrus (i.e. aerosol mass concentration below a certain threshold) by exploiting the MOCAGE aerosol forecast model.
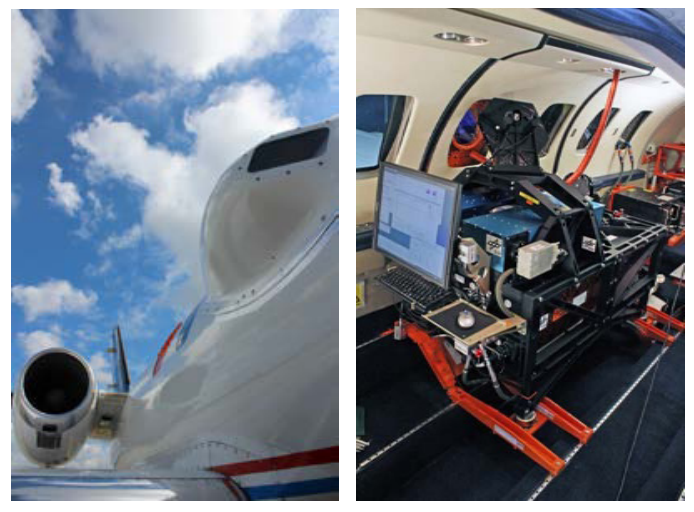

Figure 4: Left: View on the outside starboard fuselage with the fairing for laser beam transmission and reception. Right: Complete lidar and beam steering system with operator HMI.

In total, 11 flights with over 30 flight hours have been performed whereof 15 hours of lidar operation $(\approx 175 \mathrm{~Gb}$ of data). Over the whole campaign, the lidar system has been working reliably without failures, constantly delivering high output power and strong, low-noise backscatter signal beyond ranges of $15 \mathrm{~km}$.

\section{FLIGHT TEST RESULTS}

As a first step in data analysis, all data (i.e. aircraft, beam steering and lidar data) have been combined in order to identify the sections usable for scientific exploitation. For this purpose, ONERA developed a series of filters [4] to be applied first on the aircraft data (for maneuver exclusion, e.g.) and then on the lidar data (for aerosol contamination exclusion). The validated data were distributed to the concerned project partners for further evaluation. Generally, the CAT yield was rather low (as confirmed by aircraft in-situ measurements) exhibiting only "light" intensity. This encountered intensity was below the designed detection threshold nevertheless, with advanced processing techniques, respective correlations between in-situ measured turbulence (by vertical acceleration, e.g.) and lidar measurements could be shown. Going beyond the scope of this communication, we will here focus on the lidar performance itself. 
This performance evaluation is carried out by analyzing "zero" measurements, i.e. backscatter measurements in clear, smooth (non-turbulent), and aerosol-devoid air. We should recall here that the key for resolving the turbulence-induced air density fluctuations is a substantial averaging process. Therefore, the noise characteristics of the receiving and detection chain have to be evaluated. Only random "white" noise leads to a reduction of the dispersion of the mean of a measurement with the square root of the number of averaged data points.

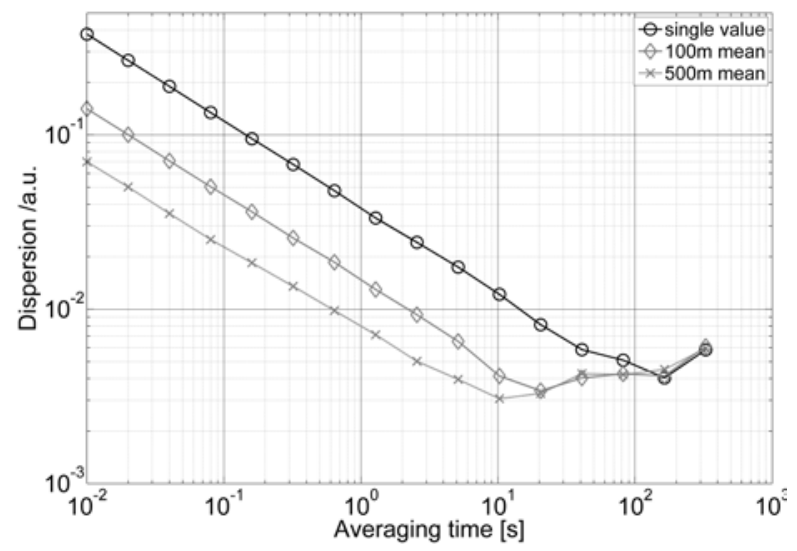

Figure 5: Stability of the backscatter measurement at $10 \mathrm{~km}$ distance for different integration times (at $100 \mathrm{~Hz}$ pulse rate) and spatial averages (at FL250).

From the campaign data, different "zero" domains have been selected (temporally close to light CAT encounters) and analyzed by employing the "time variance", a modified Allan variance. This tool basically acts as a robust and conservative estimator of the dispersion of an average - over the whole set of data. Fig. 5 shows such a plot for a $10 \mathrm{~km}$ distance: The sub-percent level, needed for moderate turbulence detection, is attained by averaging 1400 single data points ( $5 \mathrm{~m}$ resolution), i.e. $14 \mathrm{~s}$ long, or by 64 merged $500 \mathrm{~m}$ - bins, integrating less than a second. Since CAT is effective on aircraft on rather medium (tens to hundreds meters) scales, short integration times are possible. Further, longer ranges than 10 or $15 \mathrm{~km}$ may be reached since the detection system exhibits white noise behavior up to some tens of seconds. This analysis shows the suitability of such a lidar system to effectively retrieve the density fluctuations occurring in clear air turbulence.

\section{CONCLUSION}

Within the European DELICAT project, a lidar system for the airborne remote detection of CAT was designed, built, tested and evaluated by DLR and partners. The system showed superior signal to noise and stability characteristics in order to remotely resolve the air density fluctuations that are caused by CAT. Moderate turbulence at cruise altitudes may thus be detected at least 10 to $15 \mathrm{~km}$ ahead with the present system. This activity validates the application of lidar technology for improving aeronautics safety by the mitigation of CAT encounter.

\section{ACKNOWLEDGEMENTS}

The authors would like to thank the numerous colleagues from NLR, THALES, Warsaw University, Méteo France and DLR that contributed to the preparation and execution of the DELICAT flight tests. The research leading to these results has received funding from the European Union Seventh Framework Programme FP7/2007-2013 under grant agreement $n^{\circ} 233801$.

\section{REFERENCES}

[1] Feneyrou, P., J. Lehureau, and H. Barny, "Performance evaluation for long-range turbulence detection using ultraviolaet lidar," Applied Optics, 48, no. 19, pp. 3750-3759, 2009.

[2] Vrancken, P., M. Wirth, D. Rempel, G. Ehret, A. Dolfi-Bouteyre, L. Lombard, L., T. Gaudo, D. Rees, H. Barny, Ph. Rondeau, and F. Dalaudier, "Clear air turbulence detection and characterisation in the DELICAT airborne lidar project." In 25th International Laser Radar Conference (ILRC), 2010.

[3] Wirth, M., A. Fix, P. Mahnke, H. Schwarzer, F. Schrandt, and G. Ehret, "The airborne multiwavelength water vapor differential absorption lidar WALES: system design and performance," Applied Physics B: Lasers and Optics, 96, no. 1, pp. 201-213, 2009.

[4] Veerman, H.P., P. Vrancken, and L. Lombard, "Flight Testing DELICAT - A Promise for Medium-Range Clear Air Turbulence Protection", In European SETP and SFTE Flight Test Symposium, 2014. 